\title{
Diagnostic Value of Electrocardiogram in Predicting Exaggerated Blood Pressure Response to Exercise Stress Testing
}

\author{
Ali Eshraghi ${ }^{1}$, Reyhaneh Takalloo Ebdali ${ }^{2}$, Seyed Sajed Sajjadi ${ }^{2}$, Reza Golnezhad ${ }^{2}$
}

${ }^{1}$ M.D., Assistant Professor, Department of Cardiology, Atherosclerosis Prevention Research Center, Imam Reza Hospital, Mashhad University of Medical Sciences, Mashhad, Iran

${ }^{2}$ M.D., Resident of Cardiology, Department of Cardiology, Faculty of Medicine, Imam Reza Hospital, Mashhad University of Medical Sciences, Mashhad, Iran

\section{Type of article: Original}

\begin{abstract}
Introduction: It is believed that an exaggerated blood pressure response (EBPR) to exercise stress test is associated with a higher risk of cardiovascular events. It is also assumed that QT dispersion (QT-d), which was originally proposed to measure the spatial dispersion of ventricular recovery times, may have a relationship to cardiovascular events. The objective of this study was to examine the difference of changes in QT-d, Maxi-QT, Mini-QT, and QT-c (corrected QT interval) of the electrocardiogram in two groups of patients with exaggerated blood pressure responses (EBPR group) and normal responses (control group) to exercise testing. Also, the diagnostic value of each of these criteria in the prediction of EBPR was studied.

Methods: This cross-sectional study was conducted from May 2015 to February 2016 on patients suspected of coronary artery disease (CAD) undergoing exercise testing who had been referred to Ghaem and Imam Reza hospitals in Mashhad (Iran). All patients underwent a treadmill exercise test with the 12-lead ECG, which was optically scanned and digitized for analysis of QT-d, QT max, and QT min. Patients were divided into two groups of normal and EBPR to exercise testing. QT changes of ECG were compared between the two groups, and the diagnostic accuracy of QT variables for prediction of EBPR to exercise testing was studied. A multiple linear regression analysis (MLR), Pearson Chi-qquare, independent samples t-test, and receiver operating characteristic (ROC) curve were used as statistical methods in IBM SPSS version 19.

Results: Sixty patients (55\% male) with a mean age of $50.48 \pm 10.89$ years were studied in two groups of normal $(\mathrm{n}=30)$ and exaggerated blood pressure response $(\mathrm{n}=30)$ to exercise testing. Maximum QT and QT dispersion were statistically different in individuals' exaggerated blood pressure response to exercise stress test ( $\mathrm{p}<0.05)$. The logistic regression analysis revealed that none of our parameters predicted the EBPR. The ROC curve showed that 50 and 345 milliseconds for QT dispersion and Maxi-QT were the optimal cut-off points for the prediction of EBPR.

Conclusion: It seems that Maxi-QT and QT-d may be predictors of EBPR during exercise testing. Also, a significant difference in maxi-QT and QT-d was observed between two groups of patients with normal and EBPR during the exercise testing.

Keywords: Exaggerated blood pressure response, QT dispersion, Exercise stress test, EBPR exaggerated blood pressure response, QT dispersion, Exercise stress test, EBPR
\end{abstract}

\section{Introduction}

High blood pressure is considered a major risk factor for myocardial infarction, stroke, and heart and renal failures (1). According to the latest published data in 2014, the estimated prevalence of high blood pressure was $26.21 \%$ in Iran; according to Mashhad's study results of 9762 participants, a 35\% prevalence for pre-hypertension and hypertension was reported (1-3). In recent decades, increased awareness of high blood pressure has led to serious

\section{Corresponding author:}

Assistant Professor Dr. Ali Eshraghi, Department of Cardiology, Faculty of Medicine, Imam Reza Hospital, Mashhad University of Medical Sciences, Mashhad, Iran. Tel: +98.9151100754, Email: EshraghiA@mums.ac.ir Received: March 02, 2016, Accepted: May 18, 2016, Published: August 2016 iThenticate screening: May 17, 2016, English editing: July 25, 2016, Quality control: August 04, 2016

(C) 2016 The Authors. This is an open access article under the terms of the Creative Commons Attribution-NonCommercialNoDerivs License, which permits use and distribution in any medium, provided the original work is properly cited, the use is non-commercial and no modifications or adaptations are made. 
and effective efforts to control blood pressure in some European countries as well as America (1-3). Given the importance of the association between blood pressure and cardiovascular disease, many efforts have been carried out to properly screen and treat high blood pressure. Today, the measurement of blood pressure during exercise is used as a rapid predictive test to evaluate cardiovascular changes as such a measurement is highly sensitive and specific (4-6). An electrocardiogram (ECG) is also one of the most accessible and inexpensive methods for screening and checking for heart diseases; changing various parameters in this diagnostic modality can be an expression of many heart problems, and criteria such as changes in QRS and T waves, changes in intervals such as PR and QT, and criteria of QT dispersion (QT-d) and corrected QT interval (QT-c) can provide very valuable information about the heart for physicians. QT dispersion calculated by subtracting the Mini-QT from the Maxi-QT can show the overall duration of electrical activity and ventricular myocardium rest, and these criteria have been introduced in some studies as independent risk factors for sudden death based on various mechanisms, such as an increased risk of arrhythmias (4-6). The main objective of this project was, first, to examine the relationship between changes in QT$\mathrm{d}$ and other criteria, such as Maxi-QT, Mini-QT, and QT-c (corrected QT interval), within ECGs with exaggerated blood pressure responses to exercise testing; in the next phase, the poroject evaluated the diagnostic value of each of these criteria to predict the occurrence of exaggerated blood pressure response to exercise testing in patients suspected of having coronary artery diseases.

\section{Material and Methods}

\subsection{Research design and population}

This cross-sectional study was conducted from May 2015 to February 2016 on patients suspected of having coronary artery disease (CAD) undergoing exercise testing who had been referred to Ghaem and Imam Reza teaching hospitals in Mashhad, Iran. We chose Ghaem and Imam Reza hospitals because they are the main teaching and referring hospitals for patients with high blood pressure in Mashhad.

\subsection{Sampling}

The sample size of the study was estimated to be 60 patients in two groups of 30 based on the results obtained in Portas et al. (7) with respect to alpha $=5 \%$ and beta $=20 \%$. Patients were selected in a non-random and purposive manner from the primary statistical population and assigned to two EBPR and control groups according to blood pressure response to exercise testing.

\subsection{Selection criteria}

Patients with a previous history of high blood pressure, who were taking medications for cardiovascular disease, and who had electrocardiographic and pathologic findings, including ischemia, previous infarction, branch block, atrial fibrillation (AF) arrhythmia, sick sinus syndrome (SSS), and ventricular pre-excitation, were excluded from the study. The selection criteria were the same in both studied groups.

\subsection{Exercise testing}

Individuals with exaggerated blood pressure response (EBPR) during exercise testing were considered as the EBPR group, and those with normal blood pressure response to exercise testing were assigned to the control group. Age, gender, body mass index, history of smoking, dyslipidemia, and diabetes of patients were recorded at the beginning of the study. In the present study, exercise testing was carried out using a treadmill equipped with an electrocardiogram, and standard Bruce protocol was used for EST. The tests continued to gain maximum heart rate adjusted for age. The average systolic and diastolic blood pressures were recorded noninvasively using a digital sphygmomanometer before the start of testing in a sitting position, during the last 30 seconds of each stage of three minutes of exercise, and in the third minute of recovery phase in a sitting position. In this study, EBPR was defined based on one of the following three conditions during exercise testing:

1) Systolic blood pressure over $200 \mathrm{~mm} \mathrm{Hg}$

2) Increased diastolic blood pressure to the amount of over $100 \mathrm{~mm} \mathrm{Hg}$

3) Diastolic blood pressure increasing to over $10 \mathrm{~mm} \mathrm{Hg}$

\subsection{Electrocardiogram}

During the exercise, the standard 12-lead electrocardiogram of four variables, including Maxi-QT, Mini-QT, QTdispersion (QT-d), and corrected QT interval (QT-c), was recorded at the peak of exercise testing. The recording process for each of the four variables was as follows. Maxi-QT and Mini-QT were considered based on the maximum and minimum QT intervals measured in the standard 12-lead ECG, respectively. QT-d was also achieved based on the difference between the minimum and maximum values of QT-c in the standard 12-lead ECG. QT-c was 
obtained based on the division of QT by the square root of the interval between two R waves. All measurements were performed by two separate observers (specialists in heart diseases) who were unaware of the other results. In some inconsistent cases, a third observer was used as well.

\subsection{Data analysis}

Relevant statistical tables and indicators (e.g., means) were used to describe data. Continuous variables were presented as mean $\pm \mathrm{SD}$, and categorical variables were expressed as numbers and percentages. The normality of the data was analyzed using one-sample Kolmogorov-Smirnov test with modified Lilliefors, appropriate parametric methods such as an independent samples t-test were used for confirmed normality, and Mann-Whitney and Wilcoxon tests were used in the event of non-normal results. The Pearson Chi-square was used to analyze the data usng a nominal scale, and Fisher's exact test was used in cases where more than $20 \%$ of the expected frequencies in the tables were less than 5 (Cochran). Cut-off values for assessing hypertensive response to exercise and associated sensitivity and specificity were evaluated using the receiver operating characteristic (ROC) curve analysis. In addition, a multiple linear regression analysis was used to model the relationship between variables and the EBPR variable. IBM SPSS Statistics version 19 (IBM Corp., Armonk, NY, USA) was used in this study, and the significance level for all tests was less than $5 \%$.

\subsection{Ethical considerations}

Ongoing research was explained to the patient, and written consents were then obtained; information was recorded without a name using a stored code. The present study was an observational study; as there was no intervention carried out on patients, the additional risk did not cause any disease. This project was conducted under the supervision of Mashhad University of Medical Sciences (reference number 931580).

\section{Results}

\subsection{Baseline characteristics}

This study was conducted on 60 patients with suspected coronary artery disease undergoing exercise testing. Of the 60 patients under study, 33 cases were male (55\%) with a mean age of $50.48 \pm 10.89$ years. All patients underwent exercise testing and electrocardiography. Average systolic and diastolic blood pressures were measured in three steps: before exercising, at the peak of exercise, and during recovery. Based on the results, 30 patients were categorized in the EBPR group and 30 patients in the control group. The two groups had no statistically significant differences in terms of gender, but the EBPR group had a higher average age and BMI than the control group (Table 1). No statistically significant difference emerged between the two groups in terms of risk factors for smoking, diabetes, and dyslipidemia (Table 1).

\subsection{Exercise testing}

A statistically significant difference in mean systolic blood pressure occurred between the two groups in three stages: before exercising, at the peak of exercise, and during recovery. The values were higher in the EBPR group than the control group. For diastolic blood pressure, the only significant difference occurred at the peak of exercise testing, with the EBPR groups being more than the control group (Table 2).

Table 1. Comparison of demographic findings between the two groups with or without exaggerated blood pressure responses during the exercise testing

\begin{tabular}{|l|l|l|l|}
\hline Variables, Unit & $\begin{array}{l}\text { Group of exaggerated blood } \\
\text { pressure responses during } \\
\text { exercise testing }(\mathrm{n}=30)\end{array}$ & $\begin{array}{l}\text { Group of normal blood pressure } \\
\text { responses during exercise testing } \\
(\mathrm{n}=30)\end{array}$ & $\mathrm{p}$-value \\
\hline Male gender, $\mathrm{n}(\%)$ & $18(60.0 \%)$ & $15(50.0 \%)$ & 0.436 \\
\hline Age, Mean \pm SD & $54.13 \pm 8.29$ & $46.83 \pm 12.04$ & 0.008 \\
\hline Body mass index, Mean \pm SD & $26.23 \pm 3.50$ & $23.96 \pm 3.39$ & 0.014 \\
\hline History of smoking, $\mathrm{n}(\%)$ & $12(40 \%)$ & $7(23.3 \%)$ & 0.165 \\
\hline Diabetes, $\mathrm{n}(\%)$ & $9(30.0 \%)$ & $10(33.3 \%)$ & 0.891 \\
\hline Dyslipidemia, $\mathrm{n}(\%)$ & $13(43.3 \%)$ & $14(46.6 \%)$ & 0.902 \\
\hline
\end{tabular}


http://www.ephysician.ir

Table 2. Systolic and diastolic blood pressure in three phases of exercise testing

\begin{tabular}{|l|l|l|l|}
\hline Variables, Mean \pm SD & $\begin{array}{l}\text { Group of exaggerated blood } \\
\text { pressure responses during } \\
\text { exercise testing }(\mathrm{n}=30)\end{array}$ & $\begin{array}{l}\text { Group of normal blood pressure } \\
\text { responses during exercise testing } \\
(\mathrm{n}=30)\end{array}$ & p-value \\
\hline $\begin{array}{l}\text { Systolic blood pressure } \\
\text { (before exercise testing) }\end{array}$ & $126.0 \pm 12.1$ & $111.5 \pm 16.4$ & 0.000 \\
\hline $\begin{array}{l}\text { Diastolic blood pressure } \\
\text { (before exercise testing) }\end{array}$ & $73.6 \pm 5.5$ & $72.1 \pm 6.3$ & 0.432 \\
\hline $\begin{array}{l}\text { Systolic blood pressure (at the } \\
\text { peak of exercise testing) }\end{array}$ & $181.0 \pm 19.8$ & $149.00 \pm 19.92$ & 0.000 \\
\hline $\begin{array}{l}\text { Diastolic blood pressure (at } \\
\text { the peak of exercise testing) }\end{array}$ & $90.8 \pm 7.6$ & $80.3 \pm 1.8$ & 0.000 \\
\hline $\begin{array}{l}\text { Systolic blood pressure } \\
\text { (recovery) }\end{array}$ & $169.16 \pm 28.16$ & $139.00 \pm 17.87$ & 0.000 \\
\hline $\begin{array}{l}\text { Diastolic blood pressure } \\
\text { (recovery) }\end{array}$ & $83.6 \pm 14.4$ & $80.0 \pm 0.8$ & 0.999 \\
\hline
\end{tabular}

\subsection{Electrocardiogram}

The data analysis showed that the mean variables of maxi-QT and QT- $d$ in electrocardiogram were more in the EBPR group than the control group. This difference was statistically significant ( $\mathrm{p}$-value $=0.001)$. The differences in mean variables for Mini-QT and QT-c were not statistically significant between the two groups (Table 3).

Table 3. Electrocardiographic findings in patients with or without exaggerated blood pressure responses during the exercise testing

\begin{tabular}{|l|l|l|l|}
\hline $\begin{array}{l}\text { Variables, Mean } \pm \\
\text { SD }\end{array}$ & $\begin{array}{l}\text { Group of exaggerated blood pressure } \\
\text { responses during exercise testing }(\mathrm{n}=30)\end{array}$ & $\begin{array}{l}\text { Group of normal blood pressure } \\
\text { responses during exercise testing } \\
(\mathrm{n}=30)\end{array}$ & \begin{tabular}{l}
$\mathrm{p}$-value \\
\hline Maxi -QT
\end{tabular} \\
\hline Mini-QT & $366.6 \pm 24.4$ & $342.6 \pm 29.8$ & 0.001 \\
\hline QT-d & $652.0 \pm 25.2$ & $293.1 \pm 40.6$ & 0.661 \\
\hline QT-c & $383.3 \pm 50.1$ & $20.4 \pm 46.0$ & 0.001 \\
\hline
\end{tabular}

\subsection{Cut-off points of Maxi-QT and QT-d in the prediction of exaggerated blood pressure responses to stress test}

A ROC curve analysis determined the cut-off point to predict the EBPR to stress test based on the Mini-QT and QTc variables. The QT-d parameter, with a value of $50 \mathrm{~ms}(\mathrm{CI}: 95 \%(0.61-0.87))$ as the cut-off point, showed the highest sensitivity (80\%) and specificity (60\%) (Figure 1). The Maxi-QT parameter with a value of $345 \mathrm{~ms}(\mathrm{CI}$ : $95 \%$ $(0.60-0.86))$ as the cut-off point had the highest sensitivity (73\%) and specificity (64\%) (Figure 2).

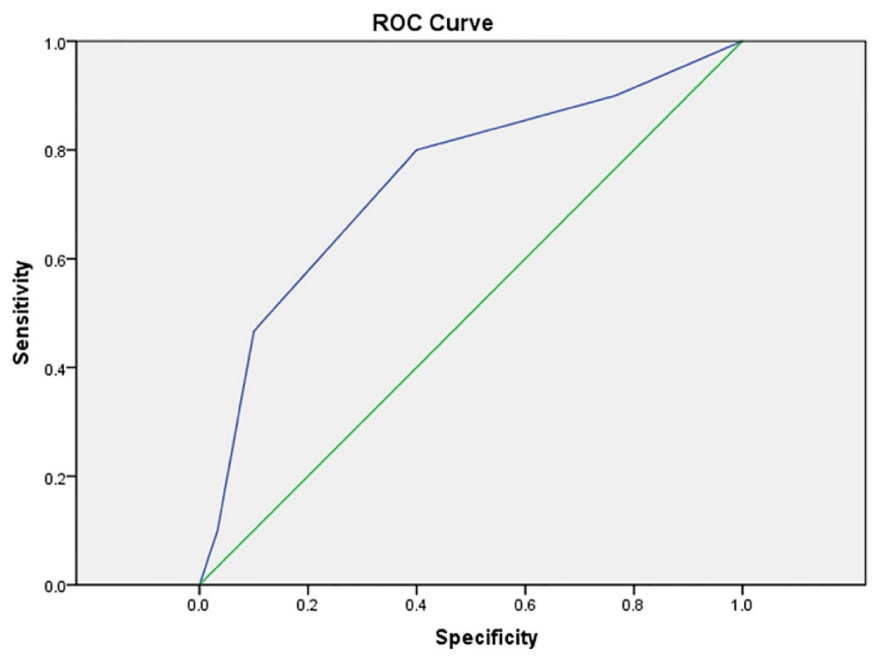

Figure 1. Investigation of cut-off point with the highest sensitivity and specificity in QT dispersion measure as a predictive factor for hypertensive response to exercise stress testing using ROC curve analysis 


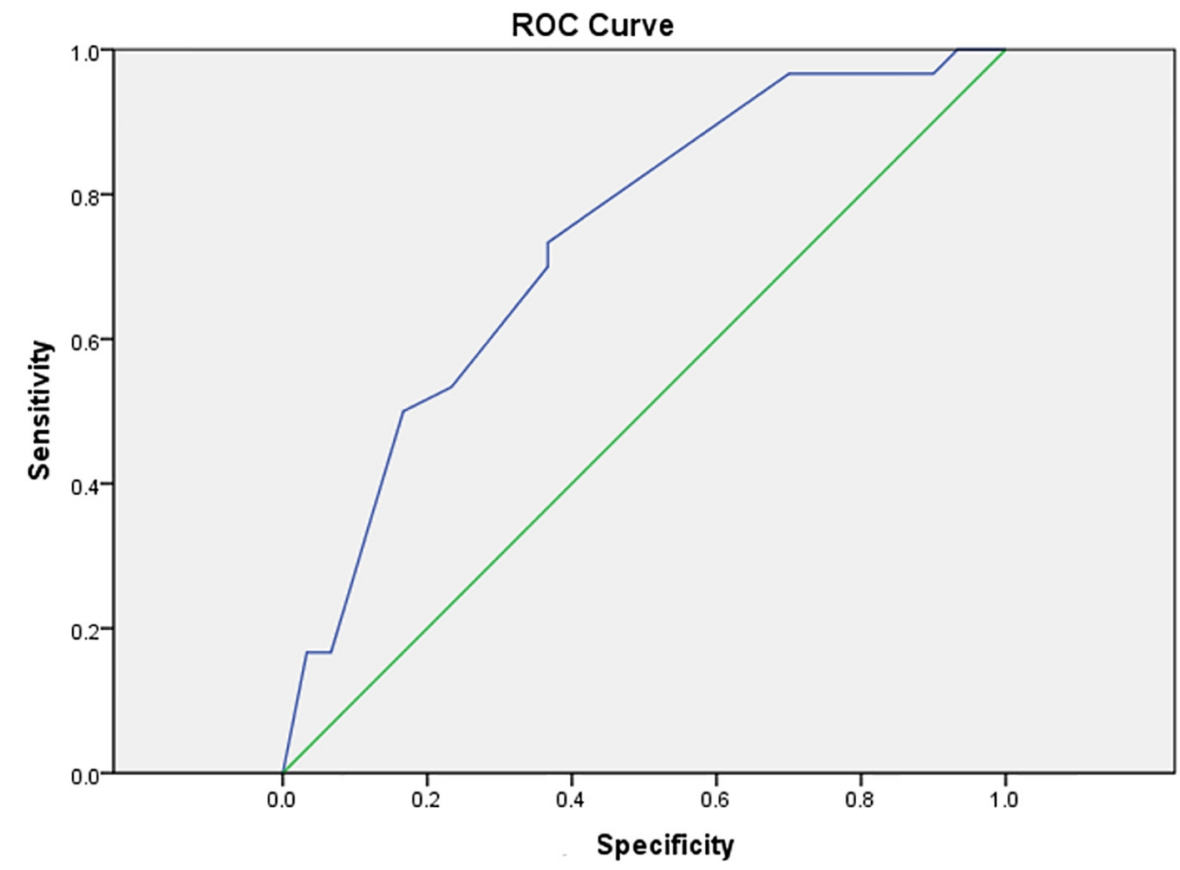

Figure 2. Investigation of cut-off point with the highest sensitivity and specificity in Maxi-QT measure as a predictive factor for hypertensive response to exercise stress testing using ROC curve analysis

\subsection{Predictive factors of exaggerated blood pressure responses to exercise testing}

The logistic regression of data demonstrated that EBPR to exercise testing are not predictable by using any of the Maxi-QT, Mini-QT, QT-d, or QT-c electrocardiographic variables or other factors such as BMI and smoking (pvalue $>0.05)$.

\section{Discussion}

In general, research has shown that EBPR to exercise testing in people with no history of high blood pressure can increase the risk of blood pressure by 2 to 3 times (8-11). Some studies have also reported associations between EBPR to exercise testing and mortality as well as cardiovascular problems, albeit generally with no described mechanism (12). Campbell Rekindle, in the late 1980s, presented the concept that the QT interval probably varied from one lead to another; in normal subjects, the QT interval may differ by $65 \mathrm{~ms}$ among varied leads (as QT dispersion). Subsequent studies raised the hypothesis that an increase in the maximum amount of the interval is likely associated with an electrical imbalance and the risk of ventricular arrhythmias (13). Few studies investigated the relationship between EBPR to exercise testing and QT dispersion, and more works have been published on the presence or absence of an association between the size of QT dispersion and risk of arrhythmia attacks $(2,14,20)$. In 1990, Lee et al. studied two groups of patients: those with long QT along with a history of ventricular arrhythmias and those taking the drug sotalol after myocardial infarction without a history of ventricular arrhythmias. The results showed that QT dispersion size was not the same in the two groups. Indeed, those with a history of ventricular arrhythmia had much more QT dispersion than the other group (14). Mantari also showed that increasing the size of QT dispersion may be one of the risk factors and predictive factors for sudden death in people, but it is not an acceptable factor in predicting a heart attack. For example, the blood pressure ratio or presence/absence of hypertension ws not mentioned in the control group. As we know that high blood pressure is a risk factor for cardiovascular events, it is possible that the difference in size of QT dispersion can result from a history of high blood pressure (2). In contrast, other studies, such as the work of Zabul et al., have found no association between QT dispersion with arrhythmias or cardiac events and mortality, but showed that other ECG criteria can have more diagnostic value. Comparing the ratio of QT-d between patients with ischemic heart attacks and those who have not experienced any such events showed that the difference was not significant between the two groups. Moreover, QT $\mathrm{d}$ was not a good measure to predict important events and fatal cardiac arrhythmia (6). In this study, the QT-c parameter was calculated and compared between the two groups, but no significant difference was found. However, the size of QT-d in the study was significantly different. Alia et al. also demonstrated that decreasing the height of the segment ST during exercise testing to more than $1 \mathrm{~mm}$, as well as correcting QT dispersion (QTDc) to over 70 
during the peak of exercise, can predict coronary problems as the independent variable (5). In comparison, our study examined the variables of smoking history, BMI, systolic and diastolic blood pressure at the peak of exercise testing and during recovery, QT-d size, QT-c size, and the maximum and minimum sizes of QT using logistic regression and found that, although the size of two criteria (i.e., Maxi-QT and QT-d) in the EBPR and control groups was significantly different, none could be predicted based on logistic regression. High blood pressure is considered one of the most serious and important risk factors in the world, and blood pressure screening programs aim to reduce cardiovascular events in many parts of the world. One searched databases to examine the relationship between QT dispersion size and EBPR during exercise testing; our research was inspired by this study in many respects. In 2013, Artas et al. studied 59 volunteer participants, 32 of whom had a history of referral to physicians due to suspected coronary disease and 27 of whom had no history of heart disease or high blood pressure (i.e., control group). These numbers are consistent with our study. Artas et al. found that EBPR to systolic blood pressure over $200 \mathrm{mmHg}$ or diastolic pressure over $100 \mathrm{mmHg}$ occurred at the peak of activity in exercise testing. EBPR were observed at the peak of exercise testing for in 32 of the 59 participants, despite having proper blood pressure in the resting position. Blood pressure was significantly higher in the EBPR group than the control group at the peak of exercise testing and during the recovery time. In addition, the maximum values of QT, QT-c, and QT-d were significantly different (pvalue $=0.001$, $p$-value $<0.001$, and $p$-value $<0.001$, respectively). In our study, although the QT-d size in the EBPR group was significantly higher than the normal response ( $\mathrm{p}$-value $=0.001$ ), no significant difference was evident in QT-c size ( $p$ - value $=0.20$ ). One reason for this difference could be the small sample size as well as the accuracy of recording in the ECG and exercise testing protocol. Many studies have used a multivariate logistic regression analysis to find the relationship between the independent variables affecting the prediction (e.g., EBPR to exercise testing in the current research). Artas et al. also used a logistic regression test to examine the predictive effects of risk factors and found that QTd and QTc intervals were significantly meaningful in predicting EBPR ( $p$-value $=0.001$ and 0.035 , respectively). However, the logistic analysis of data in our study showed that EBPR to exercise testing are not predictable with any ECG variables, including Maxi-QT, Mini-QT, QT-d, QT-c, and others such as BMI, smoking, family history of high blood pressure ( $\mathrm{p}$-value $>0.05$ ). The ROC curve analysis also revealed that a QT-d variable size greater than or equal to 40 can have the highest sensitivity $(84 \%)$ and specificity $(74 \%)$ as a diagnostic test and criterion. According to data obtained from our study, the best cut-off point was at QT-d $\geqslant 50$, when the sensitivity and specificity were $80 \%$ and $60 \%$, respectively. Another point of interest was the significant differences based on age in both the EBPR and control groups. Unfortunately, we were not able to interpret this difference due to the small sample size; although the age difference is statistically significant in both groups, both were adults with no significant difference in the number of years. Although our data are generally very similar to the data obtained in Artas et al.'s study, because of the limitations and technical differences, the results cannot be compared and interpreted. However, it may be noted as a preliminary hypothesis that blood pressure problems due to serious longterm effects on left ventricular health, such as hypertrophy, can raise the risk of cardiovascular events. Based on the studies mentioned, most protocols and criteria varied, and other factors have been thoroughly examined; there were also differences in the selected EBPR group and sampling method, and the reported criteria were not the same. All these elements led to a few studies recommending that protocols with more similarity and a larger sample size should be used in future research in order to further confirm and address shortcomings to achieve more certainty. An electrocardiogram and thorough review of relevant quantitative variables can be used as one of the easiest, most accessible, and most inexpensive screening methods, even confirming high sensitivity. One of the main limitations of this study was using the minimum number for the estimated sample size. In addition, the non-measurement of some other baseline questions in patients' medical history and medical laboratory findings is another limitation of this study.

\section{Conclusions}

According to the findings of the present research, we can benefit from ECG findings in screening patients with EBPR during exercise testing. The findings als showed that the ratio of Maxi-QT and QT-d variables further increased in the ECG of patients with EBPR during the exercise testing compared to those with normal responses, and a statistically significant relationship occurred between the type of blood pressure response to the exercise testing and ECG changes.

\section{Acknowledgments:}

We would like to acknowledge the vice chancellor for research at Mashhad University of Medical Sciences for the financial support of our study. 


\section{Conflict of Interest:}

There is no conflict of interest to be declared.

\section{Authors' contributions:}

All authors contributed to this project and article equally. All authors read and approved the final manuscript.

\section{References:}

1) Ong KL, Cheung BM, Man YB, Lau CP, Lam KS. Prevalence, awareness, treatment, and control of hypertension among United States adults 1999-2004. Hypertension. 2007; 49(1): 69-75. doi: 10.1016/j.gheart.2015.12.008. PMID: 17159087.

2) Ebrahimi M, Heidari-Bakavoli AR, Moohebati M, Mazidi M, Azarpazhooh MR, Nematy M, et al. Prevalence of Hypertension, Pre-Hypertension and Undetected hypertension in Mashhad, Iran. Acad J Sci Res. 2015; 3(5): 67-74. doi: 10.15413/ajsr.2015.0109.

3) Aghaei Meybodi HR, Khashayar P, Rezai Homami M, Heshmat R, Larijani B. Prevalence of hypertension in an Iranian population. Ren fail. 2014; 36(1): 87-91. doi: 10.3109/0886022X.2013.832315. PMID: 24028502.

4) Higham P, Campbell R. QT dispersion. British heart journal. 1994; 71(6): 508. doi: 10.1136/hrt.71.6.508. PMID: 8043327, PMCID: PMC1025441.

5) Higham PD, Furniss S, Campbell RW. QT dispersion and components of the QT interval in ischaemia and infarction. Br Heart J. 1995; 73(1): 32-6. PMID: 7888257, PMCID: PMC483752.

6) Barr CS, Naas A, Freeman M, Lang CC, Struthers AD. QT dispersion and sudden unexpected death in chronic heart failure. Lancet. 1994; 343(8893): 327-9. PMID: 7905146.

7) Ertaş F, Yavuz C, Kaya H, Karahan O, Demirtaş S, Acet H, et al. The relationship between QT dispersion and exaggerated blood pressure response to exercise stress testing. Clin Exp Hypertens. 2013; 35(6): 470-4. doi: 10.3109/10641963.2013.764891. PMID: 23387821.

8) Majahalme S, Turjanmaa V, Tuomisto $\mathrm{M}, \mathrm{Lu} \mathrm{H}$, Uusitalo A. Blood pressure responses to exercise as predictors of blood pressure level after 5 years. Am J Hypertens. 1997; 10(1): 106-16. PMID: 9008255.

9) Allison TG, Cordeiro MA, Miller TD, Daida H, Squires RW, Gau GT. Prognostic significance of exerciseinduced systemic hypertension in healthy subjects. Am J Cardiol. 1999; 83(3): 371-5. PMID: 10072226.

10) Miyai N, Arita M, Miyashita K, Morioka I, Shiraishi T, Nishio I. Blood pressure response to heart rate during exercise test and risk of future hypertension. Hypertension. 2002; 39(3): 761-6. PMID: 11897759.

11) Lima S, Albuquerque M, Oliveira J, Ayres C, Cunha J, Oliveira D, et al. Exaggerated blood pressure response during the exercise treadmill test as a risk factor for hypertension. Brazilian Journal of Medical and Biological Research. 2013; 46(4): 368-47. doi: 10.1590/1414-431X20132830.

12) Shim CY, Ha JW, Park S, Choi EY, Choi D, Rim SJ, et al. Exaggerated Blood Pressure Response to Exercise Is Associated With Augmented Rise of Angiotensin II During Exercise. J Am Coll of Cardiol. 2008; 52(4): 287-92. doi: 10.1016/j.jacc.2008.03.052. PMID: 18634984.

13) Day CP, McComb JM, Campbell RW. QT dispersion: an indication of arrhythmia risk in patients with long QT intervals. Br Heart J. 1990; 63(6): 342-4. PMID: 2375895, PMCID:PMC1024518.

14) Manttari M, Oikarinen L, Manninen V, Viitasalo M. QT dispersion as a risk factor for sudden cardiac death and fatal myocardial infarction in a coronary risk population. Heart. 1997; 78(3): 268-72. PMID: 9391289, PMCID: PMC484929.

15) De Bruyne MC, Hoes AW, Kors JA, Hofman A, Van Bemmel JH, Grobbee DE. QTe dispersion predicts cardiac mortality in the elderly the rotterdam study. Circulation. 1998; 97(5): 467-72. PMID: 9490242.

16) Zabel M, Klingenheben T, Franz MR, Hohnloser SH. Assessment of QT dispersion for prediction of mortality or arrhythmic events after myocardial infarction results of a prospective, long-term follow-up study. Circulation. 1998; 97(25): 2543-50. PMID: 9657475.

17) Hasan-Ali H, Maghraby MH, Fouad AA, Abd-Elsayed A. Corrected QT dispersion improves diagnostic performance of exercise testing in diagnosing coronary artery disease. East Mediterr Health J. 2010; 16(1): 75-81. PMID: 20214162. 